\title{
A New Analysis of Approximate Solutions for Numerical Integration Problems with Quadrature-based Methods
}

\author{
Mir Md. Moheuddin ${ }^{1, ~ *, ~ M u h a m m a d ~ A b d u s ~ S a t t a r ~ T i t u ², ~ S a d d a m ~ H o s s a i n ~}{ }^{3}$ \\ ${ }^{1}$ Department of CSE (Mathematics), Atish Dipankar University of Science and Technology (ADUST), Dhaka, Bangladesh \\ ${ }^{2}$ Department of Mathematics (General Science), Mymensingh Engineering College (MEC), Mymensingh, Bangladesh \\ ${ }^{3}$ Department of Basic Science (Mathematics), World University of Bangladesh (WUB), Dhaka, Bangladesh
}

Email address:

mirmdmoheuddinkhan@gmail.com (M. Md. Moheuddin), titumec@gmail.com (M. A. S. Titu), s.hossain656@gmail.com (S. Hossain)

${ }^{*}$ Corresponding author

\section{To cite this article:}

Mir Md. Moheuddin, Muhammad Abdus Sattar Titu, Saddam Hossain. A New Analysis of Approximate Solutions for Numerical Integration Problems with Quadrature-based Methods. Pure and Applied Mathematics Journal. Vol. 9, No. 3, 2020, pp. 46-54.

doi: 10.11648 j.pamj.20200903.11

Received: May 4, 2020; Accepted: June 9, 2020; Published: June 20, 2020

\begin{abstract}
In this paper, we mainly propose the approximate solutions to solve the integration problems numerically using the quadrature method including the Trapezoidal method, Simpson's 1/3 method, and Simpson's 3/8 method. The three proposed methods are quite workable and practically well suitable for solving integration problems. Through the MATLAB program, our numerical solutions are determined as well as compared with the exact values to verify the higher accuracy of the proposed methods. Some numerical examples have been utilized to give the accuracy rate and simple implementation of our methods. In this study, we have compared the performance of our solutions and the computational attempt of our proposed methods. Moreover, we explore and calculate the errors of the three proposed methods for the sake of showing our approximate solution's superiority. Then, among these three methods, we analyzed the approximate errors to prove which method shows more appropriate results. We also demonstrated the approximate results and observed errors to give clear idea graphically. Therefore, from the analysis, we can point out that only the minimum error is in Simpson's 1/3 method which will beneficial for the readers to understand the effectiveness in solving the several numerical integration problems.
\end{abstract}

Keywords: Numerical Integration, Trapezoidal Method, Simpson's One-Third Method, Simpson's Three-eighth's Method

\section{Introduction}

Numerical integration is the procedure of finding the approximate value of a definite integral. It is the approximate calculation of an integral with the help of numerical methods. In numerical integration, quadrature is a word that means the integration of a function that has a single variable and cubature to mean the numerical computation of multiple integrals. There are so many methods are available for numerical integration. Newton-Cotes formulas which are also known as quadrature formulas is a numerical integration technique that approximates a function $f(x)$ at a sequence of regularly spaced intervals. Numerical methods are commonly used for solving mathematical problems that are applied in science and engineering where it is difficult or even impossible to obtain exact solutions. It plays a vital role to solve mathematical problems in the numerical integration field. There are lots of numerical integral problems whose solutions cannot be achieved in closed form by using well known analytical methods, where we have to use the numerical methods to get the approximate solution of the definite integral problems under the prescribed conditions. Besides, there are various types of practical numerical methods for solving integral problems. Numerical techniques are important to evaluate the solutions of common and complicated problems easily. Nowadays the methods of numerical integrations based on information theory. Which developed to simulate information systems such as computercontrolled systems, communication systems, etc.

From the literature view, we can realize that many researchers have tried to achieve good results and accuracy quickly by using numerical methods. Mazzia et al. described and compared some numerical quadrature rules with the aim of preserving the MLPG solution accuracy and at the same 
time reducing its computational cost [1]. Different numerical rules are interpreted to count an approximate value of a definite integral to a given accuracy in the book of Davis et al., J. Douglas Fairs et al., S. S. Sastry, and L. Ridgway Scott $[2,3,4,5]$. Joulaian, M. et al. proposed a numerical approach suitable for integrating broken elements with a low number of integration points [6]. Also, Md. Jashim Uddin et al. presented the complete conception about numerical integration including Newton-Cotes formulas and compared the rate of performance or the rate of accuracy of Trapezoidal, Simpson's 1/3, and Simpson's 3/8 [7]. Jüttler, B. et al. presented a detailed case study of different quadrature schemes for isogeometric discretizations of partial differential equations on closed surfaces with Loop's subdivision scheme [8]. ur Rehman, M. et al. developed a numerical method to obtain approximate solutions for a certain class of fractional differential equations [9]. However, Thiagarajan, V. et al. demonstrated the application of AW integration scheme in the context of the Finite Cell Method which must perform numerical integration over arbitrary domains without meshing [10]. Gerry Sozio discussed a detailed summary of different techniques of numerical integration [11]. Tornabene, F. et al. have investigated and compared the accuracy and convergence behavior of two different numerical approaches based on Differential Quadrature (DQ) and Integral Quadrature (IQ) methods, respectively, when applied to the free vibration analysis of laminated plates and shells [12]. Rajesh Kumar Sinha et al. have worked to evaluate an integral polynomial discarding Taylor series [13]. Mahboubi, A. et al. presented an efficient method for automatically computing and proving bounds on some definite integrals inside the Coq formal system [14]. Yang WY et al. have explained into detail the Romberg rule, Richardson's extrapolation, Gauss quadrature, Euler method, and so forth [15]. Concepcion Ausin, M. compared various numerical integration producers and examined about more advanced numerical integration procedures [16].

What's more, Chakraborty, S. presented optimal numerical integration schemes for a family of Polygonal Finite Elements with Schwarz-Christoffel (SC) conformal mapping with improved convergence properties [17]. Chapra SC showed in Applied Numerical Methods with MATLAB to learn and apply numerical methods to solve problems in engineering and science [18]. Mettle, F. O. propounded a numerical integration method that provides improved estimates as compared to the Newton-Cotes methods of integration [19]. Smyth, G. K. focused on the process of approximating a definite integral from values of the integrand when exact mathematical integration is not available [20]. Furthermore, Marinov, T. et al. used the three basic methods which are the Midpoint, the Trapezoidal, and Simpson's rules [21]. Kwasi A. et al. proposed a numerical integration method using polynomial interpolation that provides improved estimates as compared to the Newton-Cotes methods of integration [22]. Also, Mir Md. Moheuddin et al. discussed to find out the best method through iterative methods for solving the nonlinear equation [23]. Md. Jashim
Uddin et al. presented a central difference interpolation method which is derived from the combination of Gauss's third formula, Gauss's Backward formula and Gauss's forward formula [24]. Zheng, C. et al. considered the Numerical computation of a nonlocal diffusion equation on the real axis in their paper [25].

In the present work, we discussed the numerical approximate solutions based on Newton-cotes methods for solving the definite integral problems. We have analyzed our numerical results obtained by the three proposed methods which are compared with the exact values. We have also graphically represented our discussion which obtained by our proposed methods and errors as well. Here we find a more accurate result comparing with exact results. Finally, we make our decision that Simpson's $1 / 3$ rule is very close to analytical solutions.

The paper is organized as follows: in section 2, we introduce an overview of the numerical evaluation procedures that are utilized in the next section. Section 3 shows some numerical examples to get accuracy. In section 4 we highlight the discussion of our results. Finally, section 5 includes a conclusion and recommendations of our tasks.

\section{Numerical Evaluation Procedures}

In this section we utilize three different procedures to solve numerical integration problems which are shown below:

\subsection{Procedure -1}

In numerical analysis, the trapezoidal rule or method is an idea for approximating the definite integral, the average of the left and right sums as well as usually imparts a better approximation than either does individually.

$$
I=\int_{x_{0}}^{x_{n}} f(x) d x
$$

Cotes-Newton known-well the from know we ,Also that formula quadrature general

$$
\begin{aligned}
\mathrm{I} & =h\left[n y_{0}+\frac{n^{2}}{2} \Delta y_{0}+\left(\frac{n^{3}}{3}-\frac{n^{2}}{2}\right) \frac{\Delta^{2} y_{0}}{2 !}+\left(\frac{n^{4}}{4}-n^{3}+\right.\right. \\
\left.n^{2}\right) \frac{\Delta^{3} y_{0}}{3 !} & \left.+\left(\frac{n^{5}}{5}-\frac{3 n^{4}}{2}+\frac{11 n^{3}}{3}-3 n^{2}\right) \frac{\Delta^{4} y_{0}}{4 !}+\cdots\right]
\end{aligned}
$$

Now, putting $\mathrm{n}=1$ in the above formula (1) and neglecting the second and higher difference we get,

$$
\begin{array}{r}
\int_{x_{0}}^{x_{0}+h} y d x=\mathrm{h}\left[y_{0}+\frac{1}{2} \Delta y_{0}\right] \\
=\mathrm{h}\left[y_{0}+\frac{1}{2}\left(y_{1}-y_{0}\right)\right] \\
=\frac{1}{2} h\left[\left(y_{0}+y_{1}\right)\right] \\
\text { Similarly, } \int_{x_{0+h}}^{x_{0}+2 h} \mathrm{y} \mathrm{dx}=\frac{1}{2} h\left(y_{1}+y_{2}\right)
\end{array}
$$




$$
\int_{x_{0}+(n-1) h}^{x_{0}+n h} y d x=\frac{1}{2} h\left(y_{n-1}+y_{n}\right)
$$

Adding these all integrals, we get,

$$
\int_{x_{0}}^{x_{0}+n h} y d x=\frac{h}{2}\left[y_{0} 2\left(y_{1}++y_{2}+y_{3} \ldots \ldots . .+y_{n-1}\right)+y_{n}\right]
$$

This rule is acquainted as the trapezoidal rule.

\subsection{Procedure -2}

In numerical integration, the Simpson's $1 / 3$ rule is a numerical scheme for discovering the integral $\int_{a}^{b} y d x$ within some finite limits a and $b$. Simpson's $1 / 3$ rule approximates $f$ (x) with a polynomial of degree two $\mathrm{p}(\mathrm{x})$, i.e a parabola between the two limits a and $b$, and then searches the integral of that bounded parabola which is applied to exhibit the approximate integral $\int_{a}^{b} y d x$. Besides, Simpson's one-third rule is a tract of trapezoidal rule therein the integrand is approximated through a second-order polynomial.

Also, Cotes-Newton known-well the from know we that formula quadrature general

$$
\begin{gathered}
\mathrm{I}=h\left[n y_{0}+\frac{n^{2}}{2} \Delta y_{0}+\left(\frac{n^{3}}{3}-\frac{n^{2}}{2}\right) \frac{\Delta^{2} y_{0}}{2 !}+\left(\frac{n^{4}}{4}-n^{3}+\right.\right. \\
\left.\left.n^{2}\right) \frac{\Delta^{3} y_{0}}{3 !}+\left(\frac{n^{5}}{5}-\frac{3 n^{4}}{2}+\frac{11 n^{3}}{3}-3 n^{2}\right) \frac{\Delta^{4} y_{0}}{4 !}+\cdots\right]
\end{gathered}
$$

Now, putting $\mathrm{n}=2$ in the formula (2) and neglecting the third and higher difference we get,

$$
\begin{gathered}
\int_{x_{0}}^{x_{0}+2 h} y d x=\mathrm{h}\left[2 y_{0}+2 \Delta y_{0}+\frac{\left(\frac{8}{3}-2\right)}{2} \Delta^{2} y_{0}\right] \\
=\mathrm{h}\left[2 y_{0}+2\left(y_{1}-y_{0}\right)+\frac{1}{3}\left(y_{2}-2 y_{1}+y_{0}\right)\right] \\
=\frac{1}{3} \mathrm{~h}\left(\mathrm{y}_{0}+4 \mathrm{y}_{1}+\mathrm{y}_{2}\right)
\end{gathered}
$$

Similarly, $\int_{x_{0}+2 h}^{x_{0}+4 h} y d x=\frac{1}{3} h\left(y_{2}+4 y_{3}+y_{4}\right)$

$$
\int_{x_{0}+(n-2\} h}^{x_{0}+n h} y d x=\frac{1}{3} h\left(y_{n-2}+4 y_{n-1}+y_{n}\right)
$$

When $\mathrm{n}$ is even.

Adding these all integrals, we obtain,

$$
\begin{gathered}
\int_{x_{0}}^{x_{0}+2 h} y d x+\int_{x_{0}+2 h}^{x_{0}+4 h} y d x+\cdots+\int_{x_{0}+(n-2\} h}^{x_{0+n h}} y d x \\
=\frac{1}{3} h\left[\left(y_{0}+y_{n}\right)+4\left(y_{1}+y_{3}+\cdots+y_{n-1}\right)+2\left(y_{2}+y_{4}\right.\right. \\
\left.\left.+\cdots+y_{n-2}\right)\right] \\
\text { Or, } \int_{x_{0}}^{x_{0}+n h} y d x=\frac{h}{3}\left[\left(\mathrm{y}_{0}+\mathrm{y}_{\mathrm{n}}\right)+4\left(\mathrm{y}_{1}+\mathrm{y}_{3}+\ldots .+\mathrm{y}_{\mathrm{n}-1}\right)+2\left(\mathrm{y}_{2}+\right.\right. \\
\left.\left.\mathrm{y}_{4}+\ldots+\mathrm{y}_{\mathrm{n}-2}\right)\right] .
\end{gathered}
$$

This formula is known as Simpson's one-third rule. If the number of sub-divisions of the interval is even then this method is only applied.

\subsection{Procedure -3}

Simpson's three-eight rule is a process for approximating a definite integral by evaluating the integrand at finitely many points and based upon a cubic interpolation rather than a quadratic interpolation. The difference is Simpson's 3/8 method applies a third-degree polynomial (cubic) to calculate the curve.

Further, Cotes-Newton known-well the from know we that formula quadrature general

$$
\begin{gathered}
\mathrm{I}=h\left[n y_{0}+\frac{n^{2}}{2} \Delta y_{0}+\left(\frac{n^{3}}{3}-\frac{n^{2}}{2}\right) \frac{\Delta^{2} y_{0}}{2 !}+\left(\frac{n^{4}}{4}-n^{3}+\right.\right. \\
\left.\left.n^{2}\right) \frac{\Delta^{3} y_{0}}{3 !}+\left(\frac{n^{5}}{5}-\frac{3 n^{4}}{2}+\frac{11 n^{3}}{3}-3 n^{2}\right) \frac{\Delta^{4} y_{0}}{4 !}+\cdots\right]
\end{gathered}
$$

Putting $\mathrm{n}=3$ in the formula (3) and neglecting all differences above the third, we get,

$$
\begin{gathered}
\int_{x_{0}}^{x_{0}+3 h} y d x=h\left[3 y_{0}+\frac{9}{2} \Delta y_{0}+\left(\frac{27}{3}-\frac{9}{2}\right) \frac{\Delta^{2} y_{0}}{2 !}+\left(\frac{81}{4}-27+\right.\right. \\
\left.9) \frac{\Delta^{3} y_{0}}{3 !}\right] \\
=\mathrm{h}\left[3 y_{0}+\frac{9}{2}\left(y_{1}-y_{0}\right)+\frac{9}{4}\left(y_{2}-2 y_{1}+y_{0}\right)+\frac{8}{3}\left(y_{3}-\right.\right. \\
\left.\left.3 y_{2}+3 y_{1-} y_{0}\right)\right] \\
\int_{x_{0}}^{x_{0}+3 h} y d x=\frac{3}{8} h\left(y_{0}+3 y_{1}+3 y_{2}+y_{3}\right)
\end{gathered}
$$

Similarly, $\int_{x_{0}+3 h}^{x_{0}+6 h} y d x=\frac{3}{8} h\left(y_{3}+3 y_{4}+3 y_{5}+y_{6}\right)$

$$
\int_{x_{0}+(n-3) h}^{x_{0}+n h} y d x=\frac{3}{8} h\left(y_{n-3}+3 y_{n-2}+3 y_{n-1}+y_{n}\right)
$$

Adding these all integrals, we get,

$\int_{x_{0}}^{x_{0}+3 h} y d x+\int_{x_{0}+3 h}^{x_{0}+6 h} y d x+\cdots+\int_{x_{0}+(n-3) h}^{x_{0}+n h} y d x=\frac{3}{8} \mathrm{~h}\left[\left(\mathrm{y}_{\mathrm{o}}\right.\right.$
$\left.\left.+\mathrm{y}_{\mathrm{n}}\right)+3\left(\mathrm{y}_{1}+\mathrm{y}_{2}+\mathrm{y}_{4}+\mathrm{y}_{5}+\ldots .+\mathrm{y}_{\mathrm{n}-1}\right)+2\left(\mathrm{y}_{3}+\mathrm{y}_{6}+\ldots+\mathrm{y}_{\mathrm{n}-3}\right)\right]$

This formula is known as Simpson's three-eighths rule.

\section{Numerical Examples}

In this section, we take into account three numerical examples in order to verify the accuracy of our propounded methods. With the help of this method, we evaluate the desired numerical approximations as well as errors to demonstrate which numerical methods converge better to exact value. we have given a more robust analysis of our proposed methods as well. All of the calculations are achieved by MATLAB R2013a software. Numerical solutions and errors are calculated and the outcomes are introduced graphically.

Example-1: we consider the numerical integration problem is $\int_{0.2}^{1.4}\left(\sin (x)-\log (x)+e^{x}\right) d x \quad$. The numerical approximations and errors using three proposed methods are represented in tables 1-2 and the graphs of the approximations solutions, as well as errors, are exhibited in 
figures 1-2.

Table 1. Numerical approximations using three methods.

\begin{tabular}{lllll}
\hline $\mathbf{N}$ & \multicolumn{3}{c}{ Approximate Solutions } & \multirow{2}{*}{ Exact Value } \\
\cline { 2 - 5 } & Trapezoidal method & Simpson's 1/3 method & Simpson's 3/8 method & 4.05094792986898 \\
\hline 1 & 4.64021247696075 & 3.09347498464050 & 3.48015935772056 & 4.05094792986898 \\
2 & 4.21973058090409 & 4.07956994888521 & 3.87715706408696 & 4.05094792986898 \\
3 & 4.12967337664764 & 3.52580542377883 & 4.06585598910850 & 4.05094792986898 \\
4 & 4.09627244867239 & 4.05511973792849 & 3.74577947456847 & 4.05094792986898 \\
5 & 4.08033460852562 & 3.71028720907983 & 3.93741587311721 & 4.05094792986898 \\
6 & 4.07151844721183 & 4.05213347073323 & 4.05299193050030 & 4.05094792986898 \\
7 & 4.06614000830470 & 3.80001125488056 & 3.86381734404776 & 4.05094792986898 \\
8 & 4.06262124164406 & 4.05140417263461 & 3.97316293577070 & 4.05094792986898 \\
9 & 4.06019506903661 & 3.85256639673980 & 4.05151028058523 & 4.05094792986898 \\
\hline 10 & 4.05845239330503 & 4.05115832156483 & 3.91665239016824 & \\
\hline
\end{tabular}

Table 2. Numerical errors using three methods.

\begin{tabular}{lllll}
\hline \multirow{2}{*}{$\mathbf{N}$} & \multicolumn{3}{c}{ Approximate Errors } & \multirow{2}{*}{ Exact Value } \\
\cline { 2 - 5 } & Trapezoidal method & Simpson's 1/3 method & Simpson's 3/8 method & 4.05094792986898 \\
\hline 1 & -0.58926454709176 & 0.95747294522847 & 0.57078857214841 & 4.05094792986898 \\
2 & -0.16878265103510 & -0.02862201901623 & 0.17379086578201 & 4.05094792986898 \\
3 & -0.07872544677865 & 0.52514250609014 & -0.01490805923952 & 4.05094792986898 \\
4 & -0.04532451880340 & -0.00417180805951 & 0.30516845530050 & 4.05094792986898 \\
5 & -0.02938667865663 & 0.34066072078914 & 0.11353205675176 & 4.05094792986898 \\
6 & -0.02057051734285 & -0.00118554086425 & -0.00204400063132 & 4.05094792986898 \\
7 & -0.01519207843572 & 0.25093667498841 & 0.18713058582121 & 4.05094792986898 \\
8 & -0.01167331177507 & -0.00045624276563 & 0.07778499409827 & 4.05094792986898 \\
9 & -0.00924713916762 & 0.19838153312917 & -0.00056235071625 & 4.05094792986898 \\
\hline 10 & -0.00750446343604 & -0.00021039169585 & 0.13429553970073 & \\
\hline
\end{tabular}

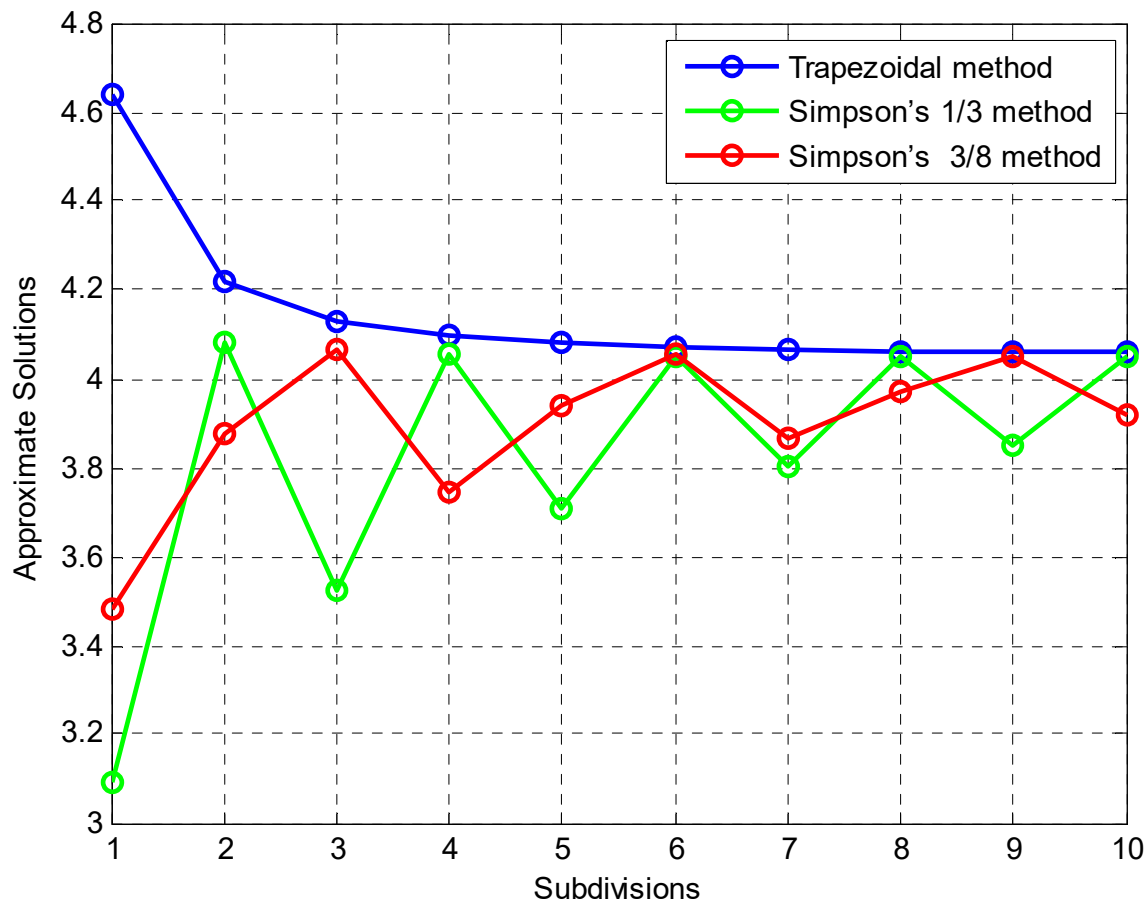

Figure 1. Observed approximate solutions for different subdivisions. 


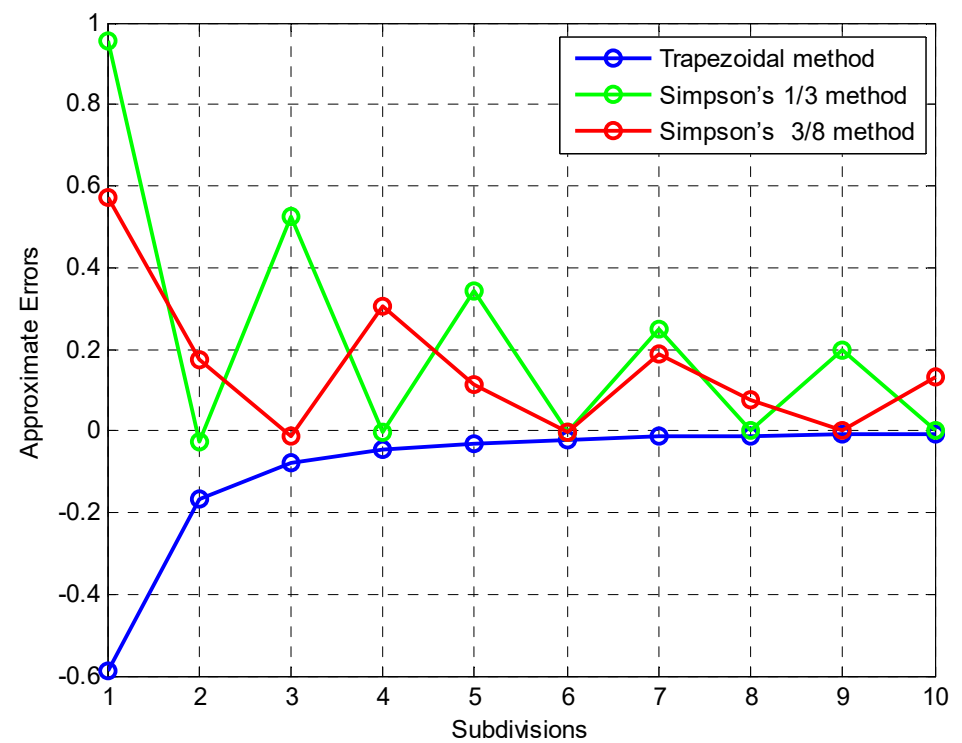

Figure 2. Observed approximate errors for different subdivisions.

Example-2: Let the numerical integration problem is $\int_{1.2}^{1.6}\left(x+\frac{1}{x}\right) d x$. The numerical approximations and errors using three proposed methods are represented in tables 3-4 and the graphs of the approximations solutions, as well as errors, are exhibited in figures $3-4$.

Table 3. Numerical approximations using three methods.

\begin{tabular}{lllll}
\hline \multirow{2}{*}{$\mathbf{N}$} & \multicolumn{3}{l}{ Approximate Solutions } & \\
\cline { 2 - 4 } & Trapezoidal method & Simpson's 1/3 method & Simpson's 3/8 method \\
\hline 1 & 0.85166666666666 & 0.56777777777777 & 0.84768210049419 & 0.84768207245178 \\
2 & 0.84869047619047 & 0.84769841269841 & 0.79508928571428 & 0.84768207245178 \\
3 & 1.15048174048174 & 0.75060606060606 & 0.84768939393939 & 0.84768207245178 \\
4 & 0.84793498168498 & 0.84768315018315 & 0.79283310439560 & 0.84768207245178 \\
5 & 0.84784399724802 & 0.78899970186905 & 0.82591616357069 & 0.84768207245178 \\
6 & 0.99751676622386 & 0.84768228762507 & 0.81614743606979 & 0.84768207245178 \\
7 & 0.84776471515601 & 0.80563300437006 & 0.83396525600450 & 0.84768207245178 \\
8 & 0.84774535101634 & 0.84768214079346 & 0.84768216817729 & 0.84768207245178 \\
9 & 0.84773207317218 & 0.81491981902041 & 0.82555470887229 & 0.84768207245178 \\
10 & 0.84772257468265 & 0.84768210049419 & & \\
\hline
\end{tabular}

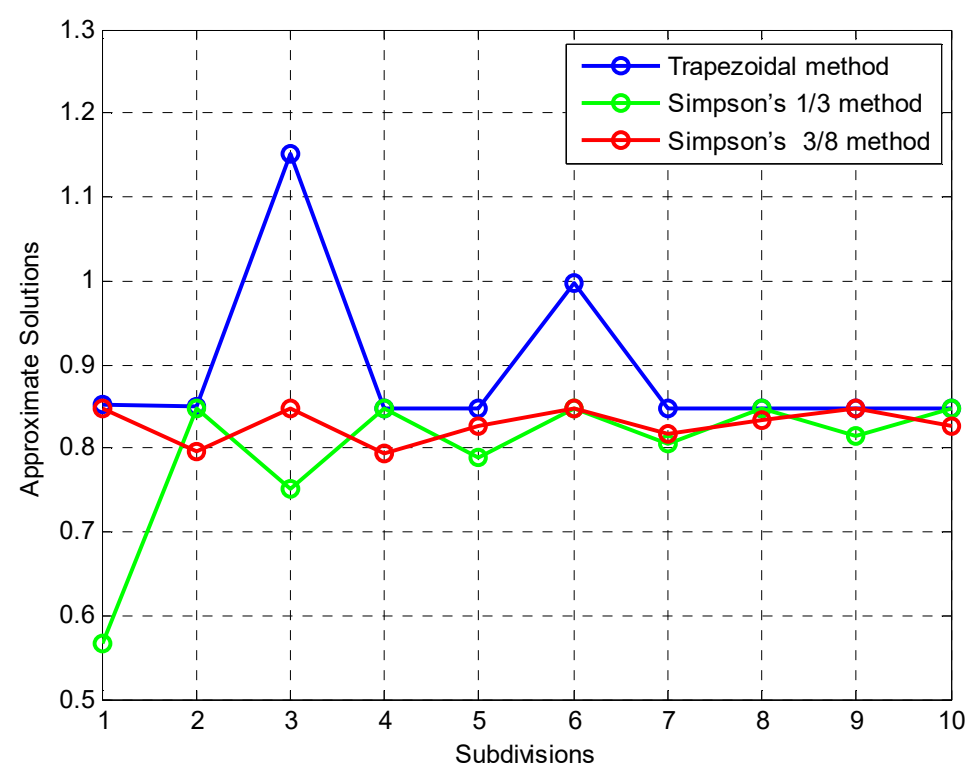

Figure 3. Observed approximate solutions for different subdivisions. 
Table 4. Numerical errors using three methods.

\begin{tabular}{|c|c|c|c|c|}
\hline \multirow{2}{*}{$\mathbf{N}$} & \multicolumn{3}{|c|}{ Approximate Errors } & \multirow{2}{*}{ Exact Value } \\
\hline & Trapezoidal method & Simpson's $1 / 3$ method & Simpson's $3 / 8$ method & \\
\hline 1 & -0.00398459421488 & 0.27990429467400 & -0.00000002804241 & 0.84768207245178 \\
\hline 2 & -0.00100840373869 & -0.00001634024663 & 0.05259278673749 & 0.84768207245178 \\
\hline 3 & -0.30279966802995 & 0.09707601184572 & -0.00000732148761 & 0.84768207245178 \\
\hline 4 & -0.00025290923320 & -0.00000107773136 & 0.05484896805617 & 0.84768207245178 \\
\hline 5 & -0.00016192479624 & 0.05868237058272 & 0.02176590888108 & 0.84768207245178 \\
\hline 6 & -0.14983469377207 & -0.00000021517329 & -0.00000048002625 & 0.84768207245178 \\
\hline 7 & -0.00008264270423 & 0.04204906808171 & 0.03153463638198 & 0.84768207245178 \\
\hline 8 & -0.00006327856456 & -0.00000006834168 & 0.01371681644727 & 0.84768207245178 \\
\hline 9 & -0.00005000072040 & 0.03276225343136 & -0.00000009572551 & 0.84768207245178 \\
\hline 10 & -0.00004050223087 & -0.00000002804241 & 0.02212736357948 & 0.84768207245178 \\
\hline
\end{tabular}

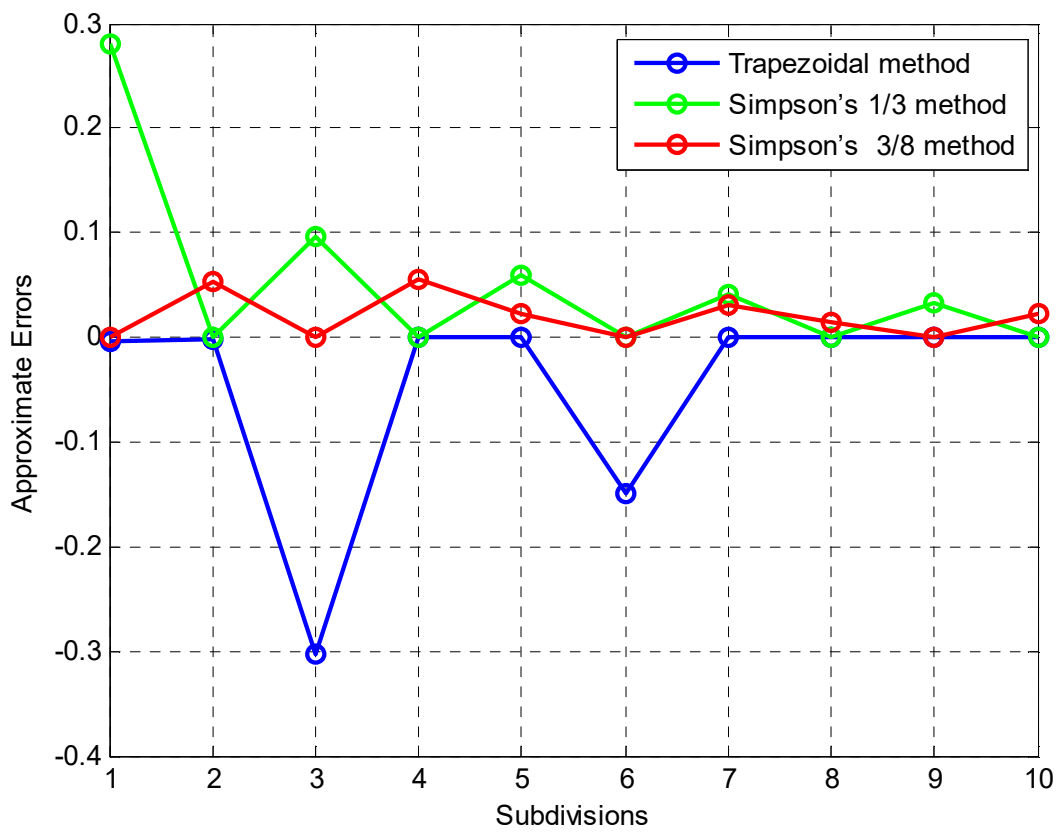

Figure 4. Observed approximate errors for different subdivisions.

Example-3: we consider the numerical integration problem is $\int_{0}^{1} \sqrt{\sin (x)+\cos (x)} d x$. The numerical approximations and errors using three proposed methods are represented in tables 5-6 and the graphs of the approximations solutions, as well as errors, are exhibited in figures 5-6.

Table 5. Numerical approximations using three methods.

\begin{tabular}{lllll}
\hline $\mathbf{N}$ & \multicolumn{3}{c}{ Approximate Solutions } & Exact Value \\
\cline { 2 - 5 } & Trapezoidal method & Simpson's 1/3 method & Simpson's 3/8 method & 1.13935597250519 \\
2 & 1.08774426638548 & 0.72516284425698 & 0.81580819978911 & 1.13935597250519 \\
3 & 1.12632558637474 & 1.13918602637116 & 1.06316423472431 & 1.13935597250519 \\
4 & 1.13355159852665 & 1.00634484351165 & 1.13927751504430 & 1.13935597250519 \\
5 & 1.13608827134762 & 1.13934249967191 & 1.06467003958875 & 1.13935597250519 \\
6 & 1.13726381074682 & 1.06013178950946 & 1.10941796814202 & 1.13935597250519 \\
7 & 1.33133642075231 & 1.13935315250086 & 1.13934991121138 & 1.13935597250519 \\
8 & 1.30446649710489 & 1.08294344801196 & 1.09700822610609 & 1.13935597250519 \\
9 & 1.13853836295454 & 1.13935506015685 & 1.12076153083630 & 1.13935597250519 \\
10 & 1.13870992145775 & 1.09555534285856 & 1.13935471182413 & 1.13935597250519 \\
\hline
\end{tabular}




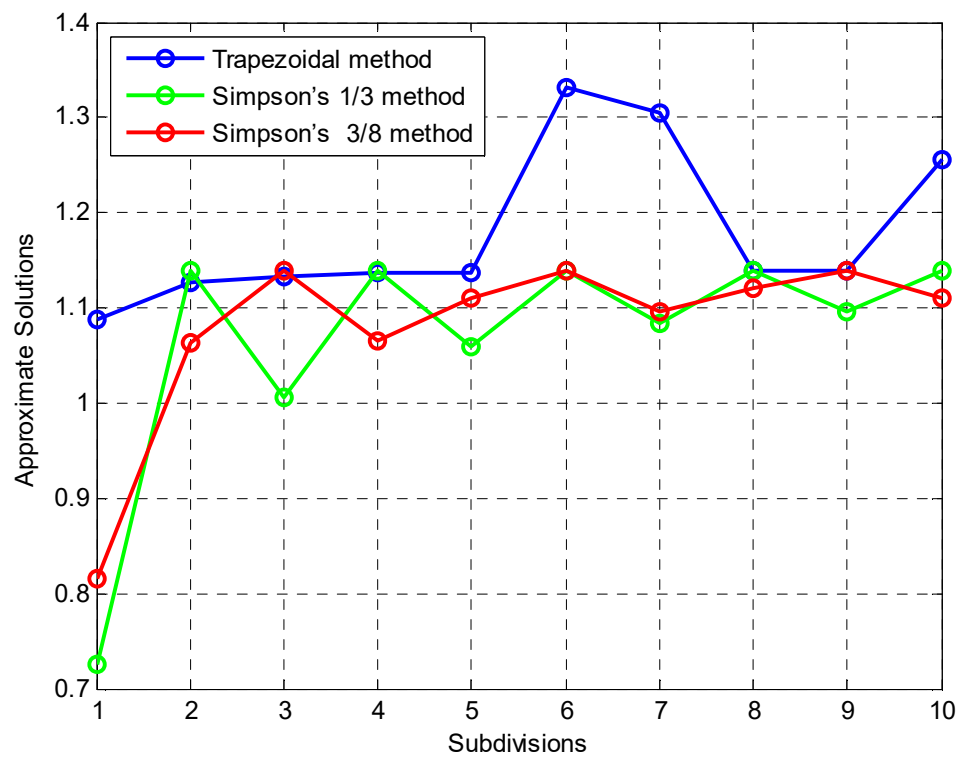

Figure 5. Observed approximate solutions for different subdivisions.

Table 6. Numerical errors using three methods.

\begin{tabular}{lllll}
\hline \multirow{2}{*}{$\mathbf{N}$} & \multicolumn{3}{c}{ Approximate Errors } & \multirow{2}{*}{ Exact Value } \\
\cline { 2 - 5 } & Trapezoidal method & Simpson's 1/3 method & Simpson's 3/8 method & 1.13935597250519 \\
\hline 1 & 0.05161170611971 & 0.41419312824820 & 0.32354777271608 & 1.13935597250519 \\
2 & 0.01303038613044 & 0.00016994613402 & 0.07619173778088 & 1.13935597250519 \\
3 & 0.00580437397853 & 0.13301112899353 & 0.00007845746089 & 1.13935597250519 \\
4 & 0.00326770115757 & 0.00001347283327 & 0.07468593291644 & 1.13935597250519 \\
5 & 0.00209216175837 & 0.07922418299573 & 0.02993800436317 & 1.13935597250519 \\
6 & -0.19198044824711 & 0.00000282000432 & 0.00000606129381 & 1.13935597250519 \\
7 & -0.16511052459969 & 0.05641252449323 & 0.04234774639910 & 1.13935597250519 \\
8 & 0.00081760955065 & 0.00000091234834 & 0.01859444166888 & 1.13935597250519 \\
9 & 0.00064605104744 & 0.04380062964662 & 0.00000126068105 & 1.13935597250519 \\
\hline
\end{tabular}

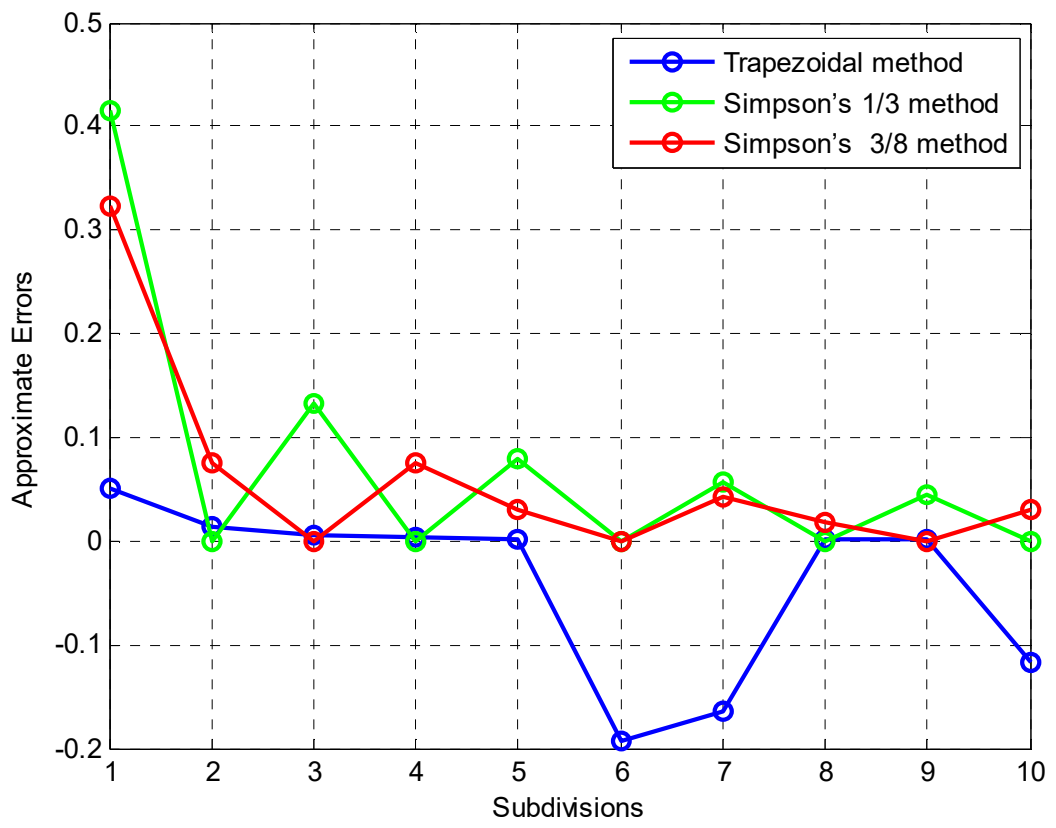

Figure 6. Observed approximate errors for different subdivisions. 


\section{Discussion of Results}

Tables 1, 3, and 5 present a comparison between approximate results and exact solution with three proposed methods and tables 2, 4, and 6 impart the numerical errors. Graphically representations for each example are shown in figures 1-2, figures 3-4, figures 5-6 where even subdivision like 2, 4, 6, 8, 10 of Simpson's 1/3 method display more correct solution and fewer errors as well. From the above tables and graphs, we can figure out that the numerical solution converges at a quicker rate only for Simpson's 1/3 method when the condition of the subdivision is even. On the other hand, approximate solution in case of other methods tends to converge slowly. Among the three proposed rules, Simpson's $1 / 3$ rule delivers very good accuracy as well as less error for each example when compared to the exact solution. Finally, we observe that Simpson's 1/3 method's solution is more accurate and appropriate than the other mentioned method's solution and Simpson's 1/3 method is the most effective method for solving definite integral problems.

\section{Conclusion and Recommendatons}

\subsection{Conclusion}

In this article, Trapezoidal, Simpson's 1/3, Simpson's 3/8 methods are used for solving integral problems numerically. To gain the desired accuracy level of numerical solutions we have taken some subdivisions sequentially where even subdivisions for Simpson's 1/3 method give good agreement in comparison with the exact values. Comparing the approximate results and errors of each problem, Trapezoidal, Simpson's 3/8 methods became less accurate because of their very slow convergence. Besides, Simpson's 1/3 methods solution is more convergent, even it is faster when compared to the other proposed methods. Lastly, it was found usually that Simpson's 1/3 method is the best useful and reliable rule in finding the approximate solutions for various integral problems.

\subsection{Recommendations}

In future research, we have the plan to develop these methods for analyzing and forecasting value among the competitors in economics, business, network systems, etc. Also, we plan to study more with numerical methods and updating our solutions with lots of analyzing factor.

\section{References}

[1] Mazzia, Annamaria, et al. A comparison of numerical integration rules for the meshless local Petrov-Galerkin method. Numerical Algorithms 45. 1-4 (2007): 61-74.

[2] Davis, Philip J., and Philip Rabinowitz. Methods of numerical integration. Courier Corporation, 2007.

[3] Douglas Fairs, J., \& L. Richard Burden, 2001, Numerical Analysis, Thomson Learning.
[4] Sastry, S. S., 2005, Introductory Methods of Numerical Analysis, Prentice-Hall of India.

[5] Ridgway Scott, L., 2011, Numerical Analysis, Princeton University Press.

[6] Joulaian, M., Hubrich, S. and Düster, A., 2016. Numerical integration of discontinuities on arbitrary domains based on moment fitting. Computational Mechanics, 57 (6), pp. 979999.

[7] Jashim Uddin Md., Moheuddin Md. Mir, and Kowsher Md. A New STUDY OF TRAPEZOIDAL, SIMPSON'S 1/3 AND SIMPSON'S 3/8 RULES OF NUMERICAL INTEGRAL PROBLEMS, Applied Mathematics and Sciences: An International Journal (MathSJ), Vol. 6, No. 4, December 2019, DOI: $10.5121 /$ mathsj.2019.6401.

[8] Jüttler, B., Mantzaflaris, A., Perl, R. and Rumpf, M., 2016. On numerical integration in isogeometric subdivision methods for PDEs on surfaces. Computer Methods in Applied Mechanics and Engineering, 302, pp. 131-146.

[9] ur Rehman, M., Idrees, A., \& Saeed, U. (2017). A quadrature method for numerical solutions of fractional differential equations. Applied Mathematics and Computation, 307, 3849 .

[10] Thiagarajan, V. and Shapiro, V., 2016. Adaptively weighted numerical integration in the finite cell method. Computer Methods in Applied Mechanics and Engineering, 311, pp. 250279.

[11] Gerry Sozio, 2009, Numerical Integration, Australian Senior Mathematics Journal, Vol-23 (1).

[12] Tornabene, F., Fantuzzi, N. and Bacciocchi, M., 2018. Strong and weak formulations based on differential and integral quadrature methods for the free vibration analysis of composite plates and shells: convergence and accuracy. Engineering Analysis with Boundary Elements, 92, pp. 3-37.

[13] Rajesh Kumar Sinha, Rakesh Kumar, 2010, Numerical method for evaluating the integrable function on a finite interval, International Journal of Engineering Science and Technology. Vol-2 (6).

[14] Mahboubi, A., Melquiond, G. and Sibut-Pinote, T., 2019. Formally verified approximations of definite integrals. Journal of Automated Reasoning, 62 (2), pp. 281-300.

[15] Yang WY, Cao W, Chung TS, Morris J. Applied numerical method using Matlab. John Wiley \& Sons, Inc. Publication; 2005.

[16] Concepcion Ausin, M. (2007) an introduction to quadrature and other numerical integration techniques, Encyclopedia of Statistics in Quality as well as reliability. Chichester, England.

[17] Chakraborty, S., S. Natarajan, S. Singh, D. Roy Mahapatra, and S. P. A. Bordas. "Optimal numerical integration schemes for a family of polygonal finite elements with SchwarzChristoffel conformal mapping." International Journal for Computational Methods in Engineering Science and Mechanics 19, no. 4 (2018): 283-304.

[18] Chapra SC (2017) Applied numerical methods with MATLAB ${ }^{\circledR}$ for engineers and scientists, 4th edition. McGrawHill Education, ISBN-13: 978-0073397962. 
[19] Mettle, F. O., Quaye, E. N. B., Asiedu, L., \& Darkwah, K. A. (2016). A Proposed Method for Numerical Integration. Journal of Advances in Mathematics and Computer Science, 17 (1), 1-15. https://doi.org/10.9734/BJMCS/2016/23048.

[20] Smyth, G. K. (2014). Numerical integration. Wiley StatsRef: Statistics Reference Online.

[21] Marinov, T., et al. (2014) Behavior of the Numerical Integration Error. Applied Mathematics, 5, 1412-1426. http://dx.doi.org/10.4236/am.2014.510133.

[22] Kwasi A., Darkwah, Ezekiel N. N., Nortey and Charles Anani Lotsi. A Proposed Numerical Integration Method Using Polynomial Interpolation. British Journal of Mathematics \& Computer Science, 16 (2): 1-11, 2016, Article no. BJMCS.25299, ISSN: 2231-0851, DOI: 10.9734/BJMCS/2016/25299.
[23] Moheuddin Md. Mir, Jashim Uddin Md. and Kowsher Md. A NEW STUDY TO FIND OUT THE BEST COMPUTATIONAL METHOD FOR SOLVING THE NONLINEAR EQUATION, Applied Mathematics and Sciences: An International Journal (MathSJ), Vol. 6, No. 2/3, September 2019, DOI: 10.5121/mathsj.2019.6302.

[24] Jashim Uddin Md., Kowsher Md. and Moheuddin Md. Mir, A NEW METHOD OF CENTRAL DIFFERENCE INTERPOLATION, Applied Mathematics and Sciences: An International Journal (MathSJ), Vol. 6, No. 2/3, September 2019, DOI: 10.5121/mathsj.2019.6301.

[25] Zheng, C., Hu, J., Du, Q. and Zhang, J., 2017. Numerical solution of the nonlocal diffusion equation on the real line. SIAM Journal on Scientific Computing, 39 (5), pp. A1951A1968. 